\title{
Extracellular Vesicle: An Emerging Mediator of Intercellular Crosstalk in Lung Inflammation and Injury
}

\author{
Heedoo Lee ${ }^{1 \dagger}$, Eric Abston ${ }^{1 \dagger}$, Duo Zhang ${ }^{1}$, Ashish Rai ${ }^{2}$ and Yang Jin ${ }^{1 *}$ \\ 'Division of Pulmonary and Critical Care Medicine, Department of Medicine, Boston University Medical Campus, Boston, \\ MA, United States, ${ }^{2}$ Department of Internal Medicine, North Shore Medical Center, Boston, MA, United States
}

Inflammatory lung responses are one of the characterized features in the pathogenesis of many lung diseases, including acute respiratory distress syndrome (ARDS) and chronic obstructive pulmonary disease (COPD). Alveolar macrophages (AMs) and alveolar epithelial cells are the first line of host defense and innate immunity. Due to their central roles in both the initiation and resolution of inflammatory lung responses, AMs constantly communicate with other lung cells, including the alveolar epithelial cells. In the past, emerging evidence suggests that extracellular vesicles play an essential role in cell-cell crosstalk. In this review, we will discuss the recent findings on the intercellular communications between lung epithelial cells and alveolar macrophages, via EV-mediated signal transfer.

Reviewed by:

Juerg Hamacher,

Lindenhof Hospital,

Switzerland

Jaya Talreja,

Wayne State University School of Medicine, United States

${ }^{*}$ Correspondence:

Yang Jin

yjin1@bu.edu

tThese authors have contributed equally to this work.

Specialty section: This article was submitted to Inflammation,

a section of the journal

Frontiers in Immunology

Received: 31 October 2017 Accepted: 13 April 2018

Published: 30 April 2018

Citation:

Lee $H$, Abston E, Zhang D, Rai A and Jin Y (2018) Extracellular

Vesicle: An Emerging Mediator of

Intercellular Crosstalk in Lung

Inflammation and Injury.

Front. Immunol. 9:924.

doi: 10.3389/fimmu.2018.00924

Keywords: macrophage-epithelium crosstalk, lung injury and inflammation, extracellular vesicles, exosome, microvesicle, apoptosis, apoptotic bodies, microRNA

\section{INTRODUCTION}

Acute respiratory distress syndrome (ARDS) and acute lung injury (ALI) is fundamentally a syndrome characterized with an intense inflammatory response, severe injury to the epithelial/endothelial barrier, and alveolar edema (1-3). Overwhelming inflammatory responses cause collateral damage in lung tissue irrespective of the initial cause, patients with ARDS universally have high levels of inflammation and circulating cytokines. However, clinical trials using anti-inflammatory agents, such as glucocorticoids to treat ARDS have failed to improve outcomes (4). Chronic obstructive pulmonary disease (COPD) is also characterized by a heterogeneous lung inflammation (5) involving epithelial cells, alveolar macrophages (AMs), neutrophils, and T cells (6). To date, the knowledge on how pulmonary cells communicate with each other and subsequently trigger an inflammatory cascade remains incompletely understood.

Alveolar macrophages are a distinct resident population that comprises the majority of inflammatory cells in the healthy lung. They form the first line of host defense against inhaled dust and/or infection, working as antigen-presenting cells and releasing powerful pro-inflammatory cytokines to drive the inflammatory response required to fight infection. Macrophages are capable to directing the type and severity of inflammatory response based on the type of injury to the lung, and also plays an important role in the resolution of inflammation and lung injury. Due to its central roles in both the initiation and resolution of inflammatory lung responses, AMs constantly communicate with other lung cells. The interactions between macrophage-neutrophil, macrophage-recruited macrophages, macrophage-lymphocyte, and macrophage-mesenchymal stem cell have been well described previously (7-10). In this review, we will discuss a novel paradigm on how macrophage-epithelial cell crosstalk occurs via extracellular vesicles (EVs) and EV-containing microRNAs (miRNAs). 
The alveolar epithelium, with their large surface area acts as the first-line defense against insult, and contribute to the integrity and function of the lungs during the development of $\operatorname{ALI}(11,12)$. Two major cell types populate the alveolar epithelium, alveolar epithelial cell type I (AECI) and type II (AECII) cells. AECII cells cover $2-5 \%$ of the surface area and have many known functions, including the secretion of surfactant $(13,14)$. AECI cells constitute the vast majority of the internal surface area (approximately 95\%) of the lung, and interact with noxious stimuli during the development of ALI $(13,14)$. Recent evidence suggests that ATI cells have important functions in innate immunity and are underappreciated players in lung cell-cell crosstalk (15).

\section{Alveolar Macrophage Polarization, Activation, and Function in the Pathogenesis of Lung Injury}

As a first-line defender, AMs are armed with high levels of pathogen-associated molecular pattern and danger-associated molecular pattern receptors in order to initiate necessary immune response (16). In response to the stimulation of microenvironmental signals, AMs often display the M1 macrophage phenotype (classically activated macrophage) or M2 phenotype (alternatively activated macrophage). M1-activated AMs produce high levels of proinflammatory cytokines, including IL- $1 \beta$, tumor necrosis factor (TNF) $-\alpha$, IL- 12 , and iNOS in the presence of IFN- $\gamma$ or IFN- $\beta$ (17). M2 macrophages produce anti-inflammatory cytokines, IL-10 and IL-1ra in response to IL-4 and IL-13. M1-activated macrophages often express MHC II (IA/IE), CD80, CD86, and CCR2, while M2-macrophages express mannose receptor, dectin-1, TfR (transferrin receptor), and CD200R (17).

During the development of ALI in animals, AMs are thought to play essential roles in both the acute inflammatory phase and resolution phase. Macrophage-derived cytokines are viewed as the major mediators. Resident AMs generate IL- 8 and TNF- $\alpha$, and subsequently stimulate neighboring cells to propagate the inflammatory responses (18). Increased BAL IL-8 level and increased IL-8 expression in AMs are associated with increased mortality in ARDS patients $(18,19)$. Additionally, macrophages secrete epithelial growth factor and GM-CSF to promote epithelial repair (20), an example of macrophage-epithelial communications. In order to achieve classical activation (M1) or alternative activation (M2), macrophages constantly receive signals from surrounding or other distant cells. A crosstalk has been reported between AMs and epithelial cells, in particular AECII cells, primarily via an autocrine and/or paracrine manner (21). The paracrine communication network between AMs and epithelial cells has been reported to affect alveolar fluid clearance in influenza virus-induced lung injury (22), via epithelial type I IFN and especially the IFN-dependent, macrophage-expressed TNF-related apoptosis-inducing ligand (TRAIL). TRAIL determines $\mathrm{Na}$, K-ATPase plasma membrane protein abundance and, thus, edema clearance during IAV infection (22). Appropriate modulation of the epithelial-macrophage crosstalk might represent a novel strategy to improve the unchecked balance of lung inflammation, epithelial damage, and fluid absorption, thus alter the outcomes in lung injury. However, the trials of cytokine suppression or antibody administration have not resulted in any favorable outcomes (23), suggesting unrecognized mechanisms that remain to be explored. For example, the minimum amount of cytokine required to maintain a concentration in the lung microenvironment, the mechanism by which the released cytokines are guided to their target cells. And how are the signaling molecules, including cytokines, proteins, and DNA/ RNAs protected from degradation or inactivation by extracellular enzymes?

Recently, emerging evidence suggest that EVs provide further understandings in addition to what we have known on the macrophage-epithelial crosstalk via cytokines and chemokines.

\section{EVs: Newly Recognized "Organelles"}

Extracellular vesicle-like molecules were initially described by Chargaff and West in 1946 (24). Currently, EVs have been isolated from almost all cell types and biological fluids, including broncho-alveolar lavage fluid (BALF). The morphology and structure of EVs can be visualized under transmission electron microscopy (Figure 1A) and 2D view (Figure 1B). In the past decade, accumulating evidence suggests that EVs play a crucial role in intercellular communication and inter-organ crosstalk.

\section{CLASSIFICATIONS, NOMENCLATURE, AND BIOGENESIS OF EVs}

According to the International Society of EVs, three main subgroups of EVs have been classified based on the size of EVs, the membrane compositions, and the mechanisms of formation(25). As illustrated in Figure 1C, apoptotic bodies (ABs) are the largest EVs and formed in the process of undergoing apoptosis. Microvesicles (MVs) are the second subgroup measuring approximately 200$500 \mathrm{~nm}$ in diameter, comprising of different sized vesicles directly protruding from plasma membranes. Exosomes are the smallest subgroup among EVs measuring approximately 30-100 nm, and are released after multiple vesicular bodies (MVBs) fuse with the plasma membrane [Figure 1C; (26)]. The mechanisms of formation of EVs are also heterogeneous. ABs are generated by cell membrane-blebbing resulting from systematic cellular breakdown during the process of apoptosis. The generation of exosomes is tightly associated with the dynamic homeostasis of endosomes/ lysosomes, trans-Golgi network, the MVBs, and intra luminal vesicles. ESCRT machinery plays an essential role in the formation of polymeric filaments and subsequently results in ILV formation, once released, called exosomes. ESCRT protein components have been confirmed in exosomes. Furthermore, ESCRT-independent mechanisms involving ceramide and tetraspanin CD63 have been reported in the exosome biogenesis and release (27-29). Fusion machinery, such as the SNARE proteins and GTPases, has been shown to regulate the ILV-plasma membrane fusion. Examples of such machineries are SNARE proteins and Rab GTPases $(30,31)$. As shown in Figure 1C, MVs are formed via the outward budding and expulsion of plasma membrane directly from the cell surface. This process of vesicle formation is often triggered by translocation of phosphatidylserine to the outer-membrane leaflet through aminophospholipid translocase activity $(32,33)$. MV formation is an energy-consuming process and requires $\operatorname{ATP}(34,35)$. 


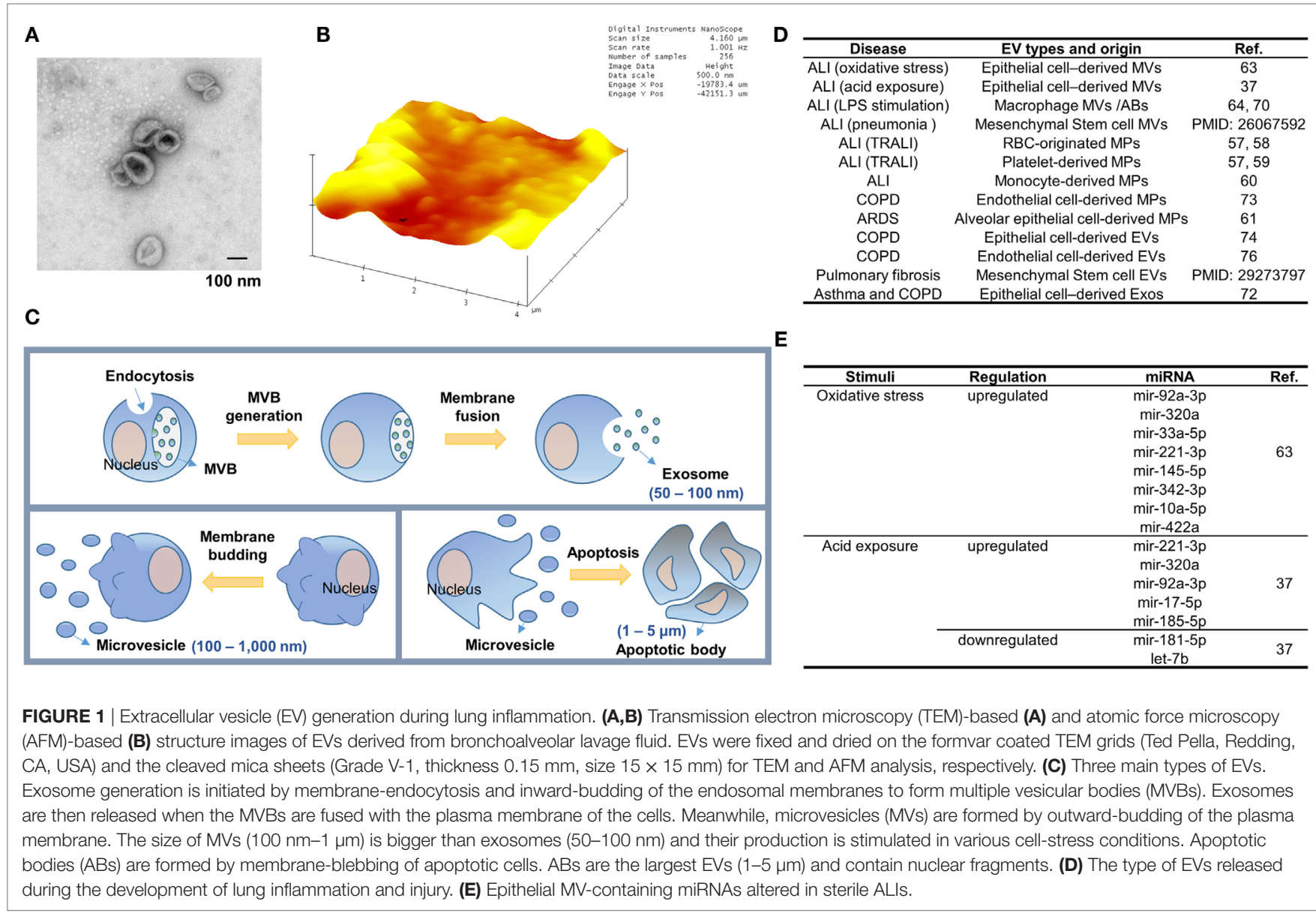

\section{EV COMPOSITIONS}

Microvesicles and exosomes are highly enriched with a variety of components and surface marker. A subset of marker proteins derived from parent cells is often detectable in EVs. Surfactant proteins, marker of alveolar type II cells (AECII), and caveolin-1, marker of alveolar type I cells (AECI) can be detected in the EVs derived from lung epithelial cells (34-37). MVs and exosomes also carry distinct proteins which can be used to differentiate the two types of EVs. Vesicle-associated membrane protein 3 can be found in the MVs while transferrin receptors are highly enriched in exosomes, but not in the MVs $(38,39)$. The marker proteins of MVs or exosomes are related to the parent cells and mechanism of secretion, thus can be used to distinguish the types of EVs, i.e., MVs vs exosomes vs ABs, as well as their origins. EV-encapsulated cytokines are a group of key proteins which potentially transmit inflammatory signals among cells. Examples of the EV-carrying cytokines include but not limit to interleukin $1 \beta$ (IL-1 $\beta$ ), IL1 $\alpha$, IL-18, macrophage migration inhibitory factor, IL-32, TNF, IL-6, vascular endothelial growth factor, IL-8 (CXCL8), fractalkine (CX3CL1), CCL2, CCL3, CCL4, CCL5, and CCL20 (40). Identifications of these important immune-modulatory cytokines/ chemokines in EVs strongly indicate that EVs carry crucial cellular functions and mediate intercellular communication.
RNAs detected in EVs generally are much smaller than cellular RNAs [less than 700 nucleotides (nt)]. Despite the smaller sizes of EV-RNAs, long non-coding RNAs, Ribosomal RNA, and the fragments of these intact RNA molecules have all been found in EVs $(26,41,42)$. A large amount of 3'UTR mRNA fragments have been identified in EVs (43). There are multiple microRNA (miRNA) binding sites on the 3'UTR mRNAs (44) and a variety of miRNAs have been identified in EVs, suggesting that EVs serve as a cargo for circulating miRNAs. However, MVs appear to be the main cargo carrying majority of miRNAs, recent studies have highlighted that there are various number of copies of "highly up-regulated" miRNAs found in tumor cells, and very low exosome detected in plasma (45).

\section{EV FUNCTIONS AND THEIR SIGNIFICANCE}

Current understanding on EVs facilitates to fill the knowledge gap on cell-cell communications. For example, EVs may partially answer the questions on how cytokines/chemokines achieve the needed concentration in the microenvironment and reach their target cells. It has been reported that cytokines are not transmitted in free forms, but appear to be associated with EVs (46). Cytokines, chemokines, protein, and miRNAs are markedly enriched inside 
EVs, suggesting that EV function as a vehicle to concentrate and transport these signaling molecules. Additionally, EVencapsulating RNAs are protected from RNaseA, and thus EVs provide a consistent source of miRNAs for therapeutic delivery and disease biomarker detection (47). EV-based drug delivery offers several advantages over conventional drug delivery systems: EVs exhibit increased stability in the blood that allows them to travel long distances within the body under both physiological and pathological conditions $(48,49)$. For example, EVs in plasma are stable up to 90 days under various storage conditions (50). In contrast, peak concentrations of TNF-alpha occur approximately $2 \mathrm{~h}$ after administration followed by a rapid decline of free TNF-alpha concentration in plasma (half-life approximately $18.2 \mathrm{~min}$ ) (5). Moreover, EVs express the same surface markers as their "mother" cells. This feature potentially provides an opportunity to deliver EV-containing molecules in a cell type-specific manner. Furthermore, EVs carrying cell type-specific markers may serve as a diagnostic agent referring as "liquid biopsy" to avoid invasive tissue diagnosis. In fact, many EV-containing molecules have been reported to potentially serve as biomarkers as shown in Figure 2A (51-54).

\section{MVs Mediate the Intercellular Crosstalk The Type of EVs Released During the Development of Lung Inflammation and Injury}

Extracellular vesicle-mediated signal transfer among lung cells is increasingly recognized as a novel mechanism by which the innate immune response is initiated (Figure 1D). A decade ago, scattered reports have shown the association between ALI and the generation of "microparticles" (MPs) derived from platelets, neutrophils, monocytes, lymphocytes, red blood cells, and endothelial and epithelial cells $(55,56)$. The MPs are now believed to be replaced with the term of MVs.

Initial observations on the potential roles of MPs were made in the transfusion-associated acute lung injury (TRALI) (57). Stored packed RBCs release MPs and these RBC-originated MPs contribute to neutrophil priming, activation, and transfusionassociated ALI (TRALI) $(57,58)$. Platelet-derived microparticles (PMPs) which carry the sCD40L increase during the storage period. PMPs may contribute to the occurrence of TRALI $(57,59)$. Furthermore, the signal transmission from monocyte/macrophage to epithelial cells has also been identified. Monocyte-derived MPs upregulate the synthesis of pro-inflammatory factors in lung epithelial cells via NF- $\kappa$ B activation through a PPAR- $\gamma$-dependent pathway (60).

Alveolar epithelial cell-derived "MPs" have been reported to be the main source of tissue factor procoagulant activity in ARDS (61). EVs detected in BALF may be derived from multiple different cell types, including but not limited to alveolar and bronchial epithelial cells, endothelial cells, AMs, neutrophils, lymphocytes fibroblasts, and the abovementioned blood cells. The type of EVs detected in BALF, i.e., MVs, exosomes, or ABs, may be dependent on the type of noxious stimuli
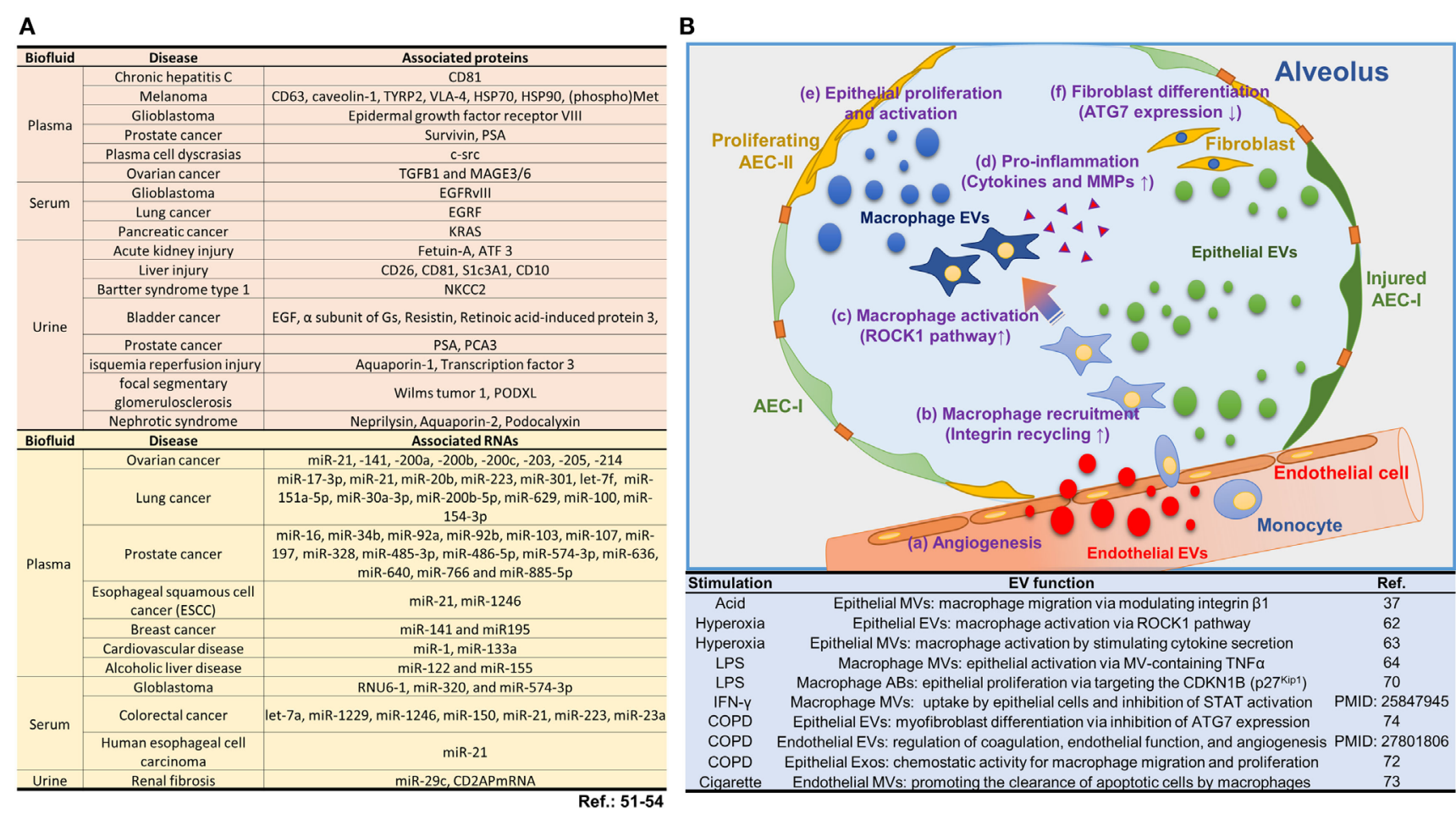

FIGURE 2 | Roles of extracellular vesicles (EVs) in lung injury. (A) EV-containing molecules reported to potentially serve as biomarkers. (B) The current reports on EVs and their functions involved in lung injury and inflammation. Schematic illustration and the summarized table for the biological pathways by which EVs contribute to the alveolar inflammatory process. 
and the severity of diseases in a time/dose-dependent dynamic manner. Moon and Lee et al. recently described that MVs form the dominant type of EVs in BALF after exposure to oxidative stress $(62,63)$. Lee et al. further reported that MVs are also the main type of EVs detected in BALF after exposure to acid inhalation (37). Approximately, $70 \%$ of BALF-EVs are MVs based on the size and marker analysis, using Nano Tracking Analysis (NTA) and Western Blot Analysis, respectively $(37,63)$. In the setting of hyperoxia or acid exposure, the second largest group of EVs is composed of exosomes, followed by a small amount of ABs. Apparently, Moon and Lee et al. focused on the early stage of exposure to noxious stimuli, whether the MVs remain dominant type of EVs after prolonged exposure to oxidative stress or acid requires further investigation. Interestingly, in LPS-induced lung injury model, a fair amount of large size vesicles are detected in BALF (64). Since the size of EVs in this study is not analyzed using NTA, the current "state of the art" analysis used in EV research, one potentially argues that the EVs studied in this report are composed of $\mathrm{AB}$ and MV mixture.

\section{The Source of MVs Detected in BALF During the Development of Lung Inflammation and Injury}

Moon et al. also report that at the usual state of mice, i.e., without exposure to noxious stimuli, AMs are the main sources of the MVs detected in BALF (62) (Figure 1D). After exposure to hyperoxiaassociated oxidative stress, MVs derived from alveolar epithelial cells increase robustly in BALF. On the other hand, AM-derived MVs remain at a steady level (62). MVs derived from other cells failed to increase as robust as the epithelial cell-derived MVs (62). Lee et al. further confirmed this observation in the setting of acidexposure induced lung injury (37). In both studies, the type of EVs was analyzed using FACS analysis via a bead-based antibody conjugation against the surface markers of interested cells, such as AMs, AECI, or AECII cells.

These studies used non-infectious or sterile stimuli (hyperoxia or acid inhalation). Alveolar epithelium has a large surface area which is exposing to the inhaled stimulators. Hyperoxia-induced oxidative stress and acid inhalation are both known to cause diffuse alveolar cell damage (65-69). Therefore, it is expected that majority of MVs in BALF are derived from lung epithelial cells. On the other hand, bacterial infection often triggers extensive pro-inflammatory responses to induce bactericidal effects. Presumably, after inhaled bacteria or LPS, the first responder which is AM may be responsible for the release of EVs into BALF. Furthermore, Moon and Lee et al. focus on the MV release in BALF at the early stage of exposure rather than prolonged treatment. All the above noxious stimuli, including both sterile and the infectious, potentially induce the generation of ABs after prolonged exposure. Zhu et al. reported recently that $\mathrm{AM}$-derived ABs exert a functional role on the epithelial cells and potentially promote epithelium proliferation (70). Their work confirmed that there is a mutual communication between epithelial cells and AMs, rather than a single direction crosstalk.

\section{The Compositions of MVs During the Development of Lung Inflammation and Injury}

Microvesicles are highly enriched with proteins, lipids, DNA, and RNA molecules $(63,71)$. Lee et al. first determine the amount of protein and RNAs in the isolated MVs. Although both components are highly upregulated in the presence of noxious stimuli, only RNA components are robustly increased in each individual MV after normalization with the number of MVs. Furthermore, Lee et al. demonstrated that small RNA molecules are elevated much more significantly than the large RNAs (63). Subsequent miRNA profiles and RT-PCR confirmation suggest that after oxidative stress, epithelial MV-containing miRNAs are dramatically altered (Figure 1E).

Functionally, Lee et al. showed that MV-miRNAs promote macrophage migration and infiltration in vitro and in vivo. After exposure to acid, epithelial MV-containing miR-17 and miR-221 exert the effects on promoting macrophage migration via modulating integrin $\beta 1$ (37). After exposure to hyperoxia, MV-containing miR-22 1 and miR-320 activate AMs by stimulating pro-inflammatory cytokine secretion (63). It appears that after specific stimuli, different MV-containing miRNAs exert specific functional roles. Collectively, in response to sterile stimuli such as hyperoxia or acid inhalation, AMs receive "pro-inflammatory" signals from the epithelial MV-containing miRNAs and subsequently respond by classical activation (M1) and increased migration.

\section{Macrophage-Derived EV-Containing miRNAs Regulate Lung Epithelial Cell Proliferation and Cell Cycle}

The EV research focused on the roles of MVs or Exos, Zhu et al. recently demonstrate that after LPS stimulation, ABs derived from macrophages exert a functional role in maintaining lung epithelial cell growth via their regulation of cell cycle (70). Although $\mathrm{AB}$ is significantly larger in size and contains more diverse contents than MV and exosome, $\mathrm{Zhu}$ et al. demonstrated that AB-containing miR-221/222 confer robust effects on promoting epithelial cell proliferation via targeting the CDKN1B $\left(\mathrm{p} 27^{\mathrm{Kip} 1}\right)$ gene $(70)$. This observation demonstrates that under certain condition, an EV-mediated macrophage-epithelium crosstalk exists in both directions, further confirming a constant and dynamic intercellular communication among different cell types in the microenvironment of lungs.

\section{EVs Play a Role in Other Inflammatory Lung Responses}

Apart from ALI and inflammation, the generation and function of EVs in the pathogenesis of other lung disease have gained increasing attention. For example, in the development of COPD, EV-mediated signaling transport has been widely reported (72-74). Epithelial cell-derived exosomes have been detected from BALF of control and asthmatic mice (72), in response to IL-13. These epithelial exosomes induce chemotaxis of undifferentiated macrophages and confer proliferative effects (72). Despite that in this report, due to the lack of NTA analysis of the sizes of EVs, the term "exosome" here may represent the three groups of EVs.

Cigarette smoke has been reported to induce the endothelial cell-derived MVs and MV-containing miRNAs, such as miR-191, miR-126, and miR125a. These miRNAs are transferred to macrophages in an EV-mediated manner, subsequently promoting 
the clearance of apoptotic cells (73). Interestingly, besides AMs, lung epithelial EVs can also transport EV-containing miRNA, miR-210, into lung fibroblasts, resulting in the inhibition of ATG7 expression and promotion of myofibroblast differentiation (74).

In addition to the EVs derived from lung epithelial cells, endothelial cells also generate a significant amount of EVs. Takahashi et al. have demonstrated that endothelial cell-derived MVs increase robustly in COPD patients compared to those in healthy volunteers (75). Furthermore, the injured endothelial cells release a significant amount of EVs, which regulate the process of coagulation, inflammation, endothelial function, and angiogenesis (76). The current reports on EVs and their functions involved in lung injury and inflammation are summarized in Figure 2B.

\section{Pitfalls and Further Directions}

Many questions remain to be answered on the role of EVs in the cell-cell crosstalk during the development of lung inflammation and injury. These questions include but are not limited to the concentration and amount of specific miRNAs in each MVs, exosomes or ABs after noxious stimuli; the effective "dose" or "amount" of MV/AB-shuttling miRNAs to trigger cellular effects;

\section{REFERENCES}

1. Martin TR. Cytokines and the acute respiratory distress syndrome (ARDS): a question of balance. Nat Med (1997) 3(3):272-3. doi:10.1038/nm0397-272

2. Matthay MA, Ware LB, Zimmerman GA. The acute respiratory distress syndrome. J Clin Invest (2012) 122(8):2731-40. doi:10.1172/JCI60331

3. Ashbaugh DG, Bigelow DB, Petty TL, Levine BE. Acute respiratory distress in adults. Lancet (1967) 2(7511):319-23. doi:10.1016/S0140-6736(67)90168-7

4. Steinberg KP, Hudson LD, Goodman RB, Hough CL, Lanken PN, Hyzy R, et al. Efficacy and safety of corticosteroids for persistent acute respiratory distress syndrome. N Engl J Med (2006) 354(16):1671-84. doi:10.1056/NEJMoa051693

5. Fabbri LM, Rabe KF. From COPD to chronic systemic inflammatory syndrome? Lancet (2007) 370(9589):797-9. doi:10.1016/S0140-6736(07)61383-X

6. Chung KF, Adcock IM. Multifaceted mechanisms in COPD: inflammation, immunity, and tissue repair and destruction. Eur Respir J (2008) 31(6):1334-56. doi:10.1183/09031936.00018908

7. Lefkowitz DL, Lefkowitz SS. Macrophage-neutrophil interaction: a paradigm for chronic inflammation revisited. Immunol Cell Biol (2001) 79(5):502-6. doi:10.1046/j.1440-1711.2001.01020.x

8. Rosenthal AS, Lipsky PE, Shevach EM. Macrophage-lymphocyte interaction and antigen recognition. Fed Proc (1975) 34(8):1743-8.

9. Mao F, Kang JJ, Cai X, Ding NF, Wu YB, Yan YM, et al. Crosstalk between mesenchymal stem cells and macrophages in inflammatory bowel disease and associated colorectal cancer. Contemp Oncol (Pozn) (2017) 21(2):91-7. doi:10.5114/wo.2017.68616

10. Mortha A, Chudnovskiy A, Hashimoto D, Bogunovic M, Spencer SP, Belkaid Y, et al. Microbiota-dependent crosstalk between macrophages and ILC3 promotes intestinal homeostasis. Science (2014) 343(6178):1249288. doi:10.1126/ science. 1249288

11. Whitsett JA, Alenghat T. Respiratory epithelial cells orchestrate pulmonary innate immunity. Nat Immunol (2015) 16(1):27-35. doi:10.1038/ni.3045

12. Nold MF, Nold-Petry CA, Zepp JA, Palmer BE, Bufler P, Dinarello CA. IL-37 is a fundamental inhibitor of innate immunity. Nat Immunol (2010) 11(11):1014-22. doi:10.1038/ni.1944

13. Gonzalez RF, Dobbs LG. Isolation and culture of alveolar epithelial type I and type II cells from rat lungs. Methods Mol Biol (2013) 945:145-59. doi:10.1007/ 978-1-62703-125-7_10

14. Castranova V, Rabovsky J, Tucker JH, Miles PR. The alveolar type II epithelial cell: a multifunctional pneumocyte. Toxicol Appl Pharmacol (1988) 93(3): 472-83. doi:10.1016/0041-008X(88)90051-8 the efficacy and pathway of MV or AB-shuttling miRNAs to enter the recipient cells. There is yet to be a study on the underlying mechanisms by which EV-shuttling miRNAs exert functions in the recipient cells.

In summary, EVs (MVs, exosomes, or ABs) play an essential role in mediating epithelial-macrophage crosstalk in the absence and presence of noxious stimuli. EV-containing miRNAs are the likely emerging targets for the development of novel therapeutic and/or diagnostic agents.

\section{AUTHOR CONTRIBUTIONS}

YJ designed, wrote, and supervised this manuscript. HL and EA wrote the manuscript, drew the schema. DZ and AR participated in the writing of the manuscript. HL and EA contributed equally to this work.

\section{FUNDING}

This work was supported by National Institutes of Health Grants R21 AI121644, R33 AI121644, R01 GM111313, R01 GM127596, and Wing Tat Lee award (all to YJ).

15. Yamamoto K, Ferrari JD, Cao Y, Ramirez MI, Jones MR, Quinton LJ, et al. Type I alveolar epithelial cells mount innate immune responses during pneumococcal pneumonia. J Immunol (2012) 189(5):2450-9. doi:10.4049/jimmunol. 1200634

16. Miyata R, van Eeden SF. The innate and adaptive immune response induced by alveolar macrophages exposed to ambient particulate matter. Toxicol Appl Pharmacol (2011) 257(2):209-26. doi:10.1016/j.taap.2011.09.007

17. Arango Duque G, Descoteaux A. Macrophage cytokines: involvement in immunity and infectious diseases. Front Immunol (2014) 5:491. doi:10.3389/ fimmu.2014.00491

18. Vlahos R, Bozinovski S. Role of alveolar macrophages in chronic obstructive pulmonary disease. Front Immunol (2014) 5:435. doi:10.3389/fimmu. 2014.00435

19. Bouros D, Alexandrakis MG, Antoniou KM, Agouridakis P, Pneumatikos I, Anevlavis S, et al. The clinical significance of serum and bronchoalveolar lavage inflammatory cytokines in patients at risk for acute respiratory distress syndrome. BMC Pulm Med (2004) 4:6. doi:10.1186/1471-2466-4-6

20. D’Angelo F, Bernasconi E, Schafer M, Moyat M, Michetti P, Maillard MH, et al. Macrophages promote epithelial repair through hepatocyte growth factor secretion. Clin Exp Immunol (2013) 174(1):60-72. doi:10.1111/cei.12157

21. Tao F, Kobzik L. Lung macrophage-epithelial cell interactions amplify particlemediated cytokine release. Am J Respir Cell Mol Biol (2002) 26(4):499-505. doi:10.1165/ajrcmb.26.4.4749

22. Peteranderl C, Morales-Nebreda L, Selvakumar B, Lecuona E, Vadasz I, Morty RE, et al. Macrophage-epithelial paracrine crosstalk inhibits lung edema clearance during influenza infection. J Clin Invest (2016) 126(4):1566-80. doi:10.1172/JCI83931

23. Marshall E. Clinical research. Lessons from a failed drug trial. Science (2006) 313(5789):901. doi:10.1126/science.313.5789.901a

24. Chargaff E, West R. The biological significance of the thromboplastic protein of blood. J Biol Chem (1946) 166(1):189-97.

25. Yanez-Mo M, Siljander PRM, Andreu Z, Zavec AB, Borras FE, Buzas EI, et al. Biological properties of extracellular vesicles and their physiological functions. J Extracell Vesicles (2015) 4:27066. doi:10.3402/jev.v4.27066

26. Crescitelli R, Lasser C, Szabo TG, Kittel A, Eldh M, Dianzani I, et al. Distinct RNA profiles in subpopulations of extracellular vesicles: apoptotic bodies, microvesicles and exosomes. J Extracell Vesicles (2013) 2:20677. doi:10.3402/ jev.v2i0.20677

27. van Niel G, Charrin S, Simoes S, Romao M, Rochin L, Saftig P, et al. The tetraspanin CD63 regulates ESCRT-independent and -dependent endosomal 
sorting during melanogenesis. Dev Cell (2011) 21(4):708-21. doi:10.1016/j. devcel.2011.08.019

28. Colombo M, Moita C, van Niel G, Kowal J, Vigneron J, Benaroch P, et al. Analysis of ESCRT functions in exosome biogenesis, composition and secretion highlights the heterogeneity of extracellular vesicles. J Cell Sci (2013) 126(Pt 24):5553-65. doi:10.1242/jcs.128868

29. Andreu Z, Yanez-Mo M. Tetraspanins in extracellular vesicle formation and function. Front Immunol (2014) 5:442. doi:10.3389/fimmu.2014.00442

30. Duman JG, Forte JG. What is the role of SNARE proteins in membrane fusion? Am J Physiol Cell Physiol (2003) 285(2):C237-49. doi:10.1152/ajpcell. 00091.2003

31. Piper RC, Katzmann DJ. Biogenesis and function of multivesicular bodies. Annu Rev Cell Dev Biol (2007) 23:519-47. doi:10.1146/annurev.cellbio.23. 090506.123319

32. Devaux PF, Herrmann A, Ohlwein N, Kozlov MM. How lipid flippases can modulate membrane structure. Biochim Biophys Acta (2008) 1778(7-8): 1591-600. doi:10.1016/j.bbamem.2008.03.007

33. Tuck S. Extracellular vesicles: budding regulated by a phosphatidylethanolamine translocase. Curr Biol (2011) 21(24):R988-90. doi:10.1016/j.cub. 2011.11.009

34. Prado N, Marazuela EG, Segura E, Fernandez-Garcia H, Villalba M, Thery C, et al. Exosomes from bronchoalveolar fluid of tolerized mice prevent allergic reaction.J Immunol (2008) 181(2):1519-25. doi:10.4049/jimmunol.181.2.1519

35. Carrasco-Ramirez P, Greening DW, Andres G, Gopal SK, Martin-Villar E, Renart J, et al. Podoplanin is a component of extracellular vesicles that reprograms cell-derived exosomal proteins and modulates lymphatic vessel formation. Oncotarget (2016) 7(13):16070-89. doi:10.18632/oncotarget.7445

36. Aliotta JM, Pereira M, Sears EH, Dooner MS, Wen S, Goldberg LR, et al. Lung-derived exosome uptake into and epigenetic modulation of marrow progenitor/stem and differentiated cells. J Extracell Vesicles (2015) 4:26166. doi: $10.3402 /$ jev.v4.26166

37. Lee H, Zhang D, Wu J, Otterbein LE, Jin Y. Lung epithelial cell-derived microvesicles regulate macrophage migration via MicroRNA-17/221-induced integrin betal recycling. J Immunol (2017) 199(4):1453-64. doi:10.4049/ jimmunol.1700165

38. Muralidharan-Chari V, Clancy J, Plou C, Romao M, Chavrier P, Raposo G, et al. ARF6-regulated shedding of tumor cell-derived plasma membrane microvesicles. Curr Biol (2009) 19(22):1875-85. doi:10.1016/j.cub.2009.09.059

39. Akers JC, Gonda D, Kim R, Carter BS, Chen CC. Biogenesis of extracellular vesicles (EV): exosomes, microvesicles, retrovirus-like vesicles, and apoptotic bodies. J Neurooncol (2013) 113(1):1-11. doi:10.1007/s11060-013-1084-8

40. Keerthikumar S, Chisanga D, Ariyaratne D, Al Saffar H, Anand S, Zhao K, et al. ExoCarta: a web-based compendium of exosomal cargo. J Mol Biol (2016) 428(4):688-92. doi:10.1016/j.jmb.2015.09.019

41. Mittelbrunn M, Sanchez-Madrid F. Intercellular communication: diverse structures for exchange of genetic information. Nat Rev Mol Cell Biol (2012) 13(5):328-35. doi:10.1038/nrm3335

42. Kogure T, Yan IK, Lin WL, Patel T. Extracellular vesicle-mediated transfer of a novel long noncoding RNA TUC339: a mechanism of intercellular signaling in human hepatocellular cancer. Genes Cancer (2013) 4(7-8):261-72. doi:10.1177/1947601913499020

43. Batagov AO, Kurochkin IV. Exosomes secreted by human cells transport largely mRNA fragments that are enriched in the 3'-untranslated regions. Biol Direct (2013) 8:12. doi:10.1186/1745-6150-8-12

44. Lee I, Ajay SS, Yook JI, Kim HS, Hong SH, Kim NH, et al. New class of microRNA targets containing simultaneous 5'-UTR and 3'-UTR interaction sites. Genome Res (2009) 19(7):1175-83. doi:10.1101/gr.089367.108

45. Chevillet JR, Kang Q, Ruf IK, Briggs HA, Vojtech LN, Hughes SM, et al. Quantitative and stoichiometric analysis of the microRNA content of exosomes. Proc Natl Acad Sci U S A (2014) 111(41):14888-93. doi:10.1073/ pnas. 1408301111

46. Konadu KA, Chu J, Huang MB, Amancha PK, Armstrong W, Powell MD, et al. Association of cytokines with exosomes in the plasma of HIV-1-seropositive individuals. J Infect Dis (2015) 211(11):1712-6. doi:10.1093/infdis/jiu676

47. Cheng L, Sharples RA, Scicluna BJ, Hill AF. Exosomes provide a protective and enriched source of miRNA for biomarker profiling compared to intracellular and cell-free blood. J Extracell Vesicles (2014) 3:23743. doi:10.3402/ jev.v3.23743
48. Jiang XC, Gao JQ. Exosomes as novel bio-carriers for gene and drug delivery. Int J Pharm (2017) 521(1-2):167-75. doi:10.1016/j.ijpharm.2017.02.038

49. Yousefpour P, Chilkoti A. Co-opting biology to deliver drugs. Biotechnol Bioeng (2014) 111(9):1699-716. doi:10.1002/bit.25307

50. Kalra H, Adda CG, Liem M, Ang CS, Mechler A, Simpson RJ, et al. Comparative proteomics evaluation of plasma exosome isolation techniques and assessment of the stability of exosomes in normal human blood plasma. Proteomics (2013) 13(22):3354-64. doi:10.1002/pmic.201300282

51. Lin J, Li J, Huang B, Liu J, Chen X, Chen XM, et al. Exosomes: novel biomarkers for clinical diagnosis. ScientificWorldJournal (2015) 2015:657086. doi:10.1155/2015/657086

52. Properzi F, Logozzi M, Fais S. Exosomes: the future of biomarkers in medicine. Biomark Med (2013) 7(5):769-78. doi:10.2217/bmm.13.63

53. Gamez-Valero A, Lozano-Ramos SI, Bancu I, Lauzurica-Valdemoros R, Borras FE. Urinary extracellular vesicles as source of biomarkers in kidney diseases. Front Immunol (2015) 6:6. doi:10.3389/fimmu.2015.00006

54. Zocco D, Ferruzzi P, Cappello F, Kuo WP, Fais S. Extracellular vesicles as shuttles of tumor biomarkers and anti-tumor drugs. Front Oncol (2014) 4:267. doi:10.3389/fonc.2014.00267

55. Barteneva NS, Fasler-Kan E, Bernimoulin M, Stern JN, Ponomarev ED, Duckett L, et al. Circulating microparticles: square the circle. BMC Cell Biol (2013) 14:23. doi:10.1186/1471-2121-14-23

56. Lacedonia D, Carpagnano GE, Trotta T, Palladino GP, Panaro MA, Zoppo LD, et al. Microparticles in sputum of COPD patients: a potential biomarker of the disease? Int J Chron Obstruct Pulmon Dis (2016) 11:527-33. doi:10.2147/ COPD.S99547

57. Xie RF, Hu P, Li W, Ren YN, Yang J, Yang YM, et al. The effect of platelet-derived microparticles in stored apheresis platelet concentrates on polymorphonuclear leucocyte respiratory burst. Vox Sang (2014) 106(3):234-41. doi:10.1111/ vox.12092

58. Belizaire RM, Prakash PS, Richter JR, Robinson BR, Edwards MJ, Caldwell CC, et al. Microparticles from stored red blood cells activate neutrophils and cause lung injury after hemorrhage and resuscitation. J Am Coll Surg (2012) 214(4):648-55; discussion 56-7. doi:10.1016/j.jamcollsurg.2011.12.032

59. Xie RF, Hu P, Wang ZC, Yang J, Yang YM, Gao L, et al. Platelet-derived microparticles induce polymorphonuclear leukocyte-mediated damage of human pulmonary microvascular endothelial cells. Transfusion (2015) 55(5):1051-7. doi:10.1111/trf.12952

60. Neri T, Armani C, Pegoli A, Cordazzo C, Carmazzi Y, Brunelleschi S, et al. Role of NF-kappaB and PPAR-gamma in lung inflammation induced by monocyte-derived microparticles. Eur Respir J (2011) 37(6):1494-502. doi:10.1183/09031936.00023310

61. Bastarache JA, Fremont RD, Kropski JA, Bossert FR, Ware LB. Procoagulant alveolar microparticles in the lungs of patients with acute respiratory distress syndrome. Am J Physiol Lung Cell Mol Physiol (2009) 297(6):L1035-41. doi:10.1152/ajplung.00214.2009

62. Moon HG, Cao Y, Yang J, Lee JH, Choi HS, Jin Y. Lung epithelial cell-derived extracellular vesicles activate macrophage-mediated inflammatory responses via ROCK1 pathway. Cell Death Dis (2015) 6:e2016. doi:10.1038/cddis.2015.282

63. Lee H, Zhang D, Zhu Z, Dela Cruz CS, Jin Y. Epithelial cell-derived microvesicles activate macrophages and promote inflammation via microvesiclecontaining microRNAs. Sci Rep (2016) 6:35250. doi:10.1038/srep35250

64. Soni S, Wilson MR, O’Dea KP, Yoshida M, Katbeh U, Woods SJ, et al. Alveolar macrophage-derived microvesicles mediate acute lung injury. Thorax (2016) 71(11):1020-9. doi:10.1136/thoraxjnl-2015-208032

65. Bhandari V, Choo-Wing R, Lee CG, Zhu Z, Nedrelow JH, Chupp GL, et al. Hyperoxia causes angiopoietin 2-mediated acute lung injury and necrotic cell death. Nat Med (2006) 12(11):1286-93. doi:10.1038/nm1494

66. Clement A, Edeas M, Chadelat K, Brody JS. Inhibition of lung epithelial cell proliferation by hyperoxia. Posttranscriptional regulation of proliferationrelated genes. J Clin Invest (1992) 90(5):1812-8. doi:10.1172/JCI116056

67. Ray P, Devaux Y, Stolz DB, Yarlagadda M, Watkins SC, Lu Y, et al. Inducible expression of keratinocyte growth factor (KGF) in mice inhibits lung epithelial cell death induced by hyperoxia. Proc Natl Acad Sci U S A (2003) 100(10):6098-103. doi:10.1073/pnas.1031851100

68. Corne J, Chupp G, Lee CG, Homer RJ, Zhu Z, Chen Q, et al. IL-13 stimulates vascular endothelial cell growth factor and protects against hyperoxic acute lung injury. J Clin Invest (2000) 106(6):783-91. doi:10.1172/JCI9674 
69. Matute-Bello G, Frevert CW, Martin TR. Animal models of acute lung injury. Am J Physiol Lung Cell Mol Physiol (2008) 295(3):L379-99. doi:10.1152/ ajplung.00010.2008

70. Zhu Z, Zhang D, Lee H, Menon AA, Wu J, Hu K, et al. Macrophage-derived apoptotic bodies promote the proliferation of the recipient cells via shuttling microRNA-221/222. J Leukoc Biol (2017) 101(6):1349-59. doi:10.1189/jlb. 3A1116-483R

71. Balaj L, Lessard R, Dai L, Cho YJ, Pomeroy SL, Breakefield XO, et al. Tumour microvesicles contain retrotransposon elements and amplified oncogene sequences. Nat Commun (2011) 2:180. doi:10.1038/ncomms1180

72. Kulshreshtha A, Ahmad T, Agrawal A, Ghosh B. Proinflammatory role of epithelial cell-derived exosomes in allergic airway inflammation. J Allergy Clin Immunol(2013) 131(4):1194-203, 1203.e1-14. doi:10.1016/j.jaci.2012.12.1565

73. Serban KA, Rezania S, Petrusca DN, Poirier C, Cao D, Justice MJ, et al. Structural and functional characterization of endothelial microparticles released by cigarette smoke. Sci Rep (2016) 6:31596. doi:10.1038/srep31596

74. Fujita Y, Araya J, Ito S, Kobayashi K, Kosaka N, Yoshioka Y, et al. Suppression of autophagy by extracellular vesicles promotes myofibroblast differentiation in COPD pathogenesis. J Extracell Vesicles (2015) 4:28388. doi:10.3402/jev. v4.28388
75. Takahashi T, Kobayashi S, Fujino N, Suzuki T, Ota C, He M, et al. Increased circulating endothelial microparticles in COPD patients: a potential biomarker for COPD exacerbation susceptibility. Thorax (2012) 67(12):1067-74. doi:10.1136/thoraxjnl-2011-201395

76. Kadota T, Fujita Y, Yoshioka Y, Araya J, Kuwano K, Ochiya T. Extracellular vesicles in chronic obstructive pulmonary disease. Int J Mol Sci (2016) 17(11):1801. doi:10.3390/ijms17111801

Conflict of Interest Statement: The authors declare that the research was conducted in the absence of any commercial or financial relationships that could be construed as a potential conflict of interest.

Copyright $\odot 2018$ Lee, Abston, Zhang, Rai and Jin. This is an open-access article distributed under the terms of the Creative Commons Attribution License (CC BY).

The use, distribution or reproduction in other forums is permitted, provided the original author(s) and the copyright owner are credited and that the original publication in this journal is cited, in accordance with accepted academic practice. No use, distribution or reproduction is permitted which does not comply with these terms. 